\title{
Lapurdum
}

Euskal ikerketen aldizkaria | Revue d'études basques |

Revista de estudios vascos | Basque studies review

$15 \mid 2011$

Numéro $X V$

\section{Compétence(s), performance et déviance(s) : questions générales et exemples basques}

\section{Georges Rebuschi}

\section{OpenEdition}

Journals

Édition électronique

URL : http://journals.openedition.org/lapurdum/2321

DOI : 10.4000/lapurdum.2321

ISSN : 1965-0655

Éditeur

IKER

Édition imprimée

Date de publication : 1 octobre 2011

Pagination : 43-63

ISSN : 1273-3830

Référence électronique

Georges Rebuschi, «Compétence(s), performance et déviance(s) : questions générales et exemples basques », Lapurdum [En ligne], 15 | 2011, mis en ligne le 15 octobre 2014, consulté le 10 décembre 2020. URL : http://journals.openedition.org/lapurdum/2321 ; DOI : https://doi.org/10.4000/lapurdum 2321 


\section{Competénces(s), performance et déviance(s): questions générales et exemples basques*}

Georges REBUSCHI

Sorbonne Nouvelle \& LACITO, associé à l'UMR 5478 (IKER)

\section{0 . Introduction}

Ce travail commence par une présentation de la position méthodologique de la linguistique chomskyenne en général (\$1) et des types de règles et déviances ou écarts usuellement recensés par les linguistes (\$2). Ensuite, l'opposition bien connue entre compétence et performance linguistiques, due à Noam Chomsky, est illustrée avec un exemple très standard (ce qu'on pourrait construire mais ne peut être compris), puis approfondie avec le cas de figure de ce qui ne peut simplement pas être construit (\$3). Deux modèles cognitivistes de performance sont alors présentés, qui ne sont pas en concurrence mais fonctionneraient de manière complémentaire selon les situations de production et d'interprétation (\$4). Enfin, en \$5, suite à l'examen d'un « monstre linguistique » dû à un grand linguiste basque qui l'a produit spontanément, quoique par écrit, on avancera l'hypothèse que les locuteurs ont en fait deux types de compétence à leur disposition, la compétence chomskyenne n'étant qu'un système en arrière-plan qui n’intervient pas nécessairement dans la production spontanée.

\section{Les niveaux d'appréhension des faits et des abstractions linguistiques}

Pour comprendre la construction de l'opposition entre compétence et performance, il est utile de considérer cinq niveaux d'appréhension de faits et de construction d'hypothèses théoriques.

1. Il y a d'abord les énoncés concrets, produits (et compris - bien ou mal) par des locuteurs spécifiques dans des situations spécifiques.

2. Viennent ensuite les phrases, i.e. les énoncés débarrassés par exemple de la référence spécifique à l'identité du locuteur et de l'interlocuteur quand des pronoms de lère et/ ou 2e personne sont employés, des effets pragmatiques comme l'injonction « Vous êtes prié de vous taire » où la valeur n'est pas descriptive, mais proche d'un impératif, cet

\footnotetext{
* Je tiens à remercier Jean-Luis Pétrissans pour m'avoir invité à Cambo, l'audience pour les questions posées, Beñat Oyharçabal pour la discussion de divers exemples, etBattittu Coyos et Marie Pourquié pour leurs remarques sur une première version de ce texte-ci. Merci également à l'équipe IKER du CNRS, qui a financé mon déplacement, et à J. et C. Haritschelhar pour leur hospitalité et leur amitié.
} 
effet ne se manifestant pas si le sujet est à la 3e personne...) : on est donc déjà dans l'abstraction'.

3. Une langue peut être définie en extension comme un ensemble ouvert de phrases — c'est déjà la définition de $\boldsymbol{a}$ language selon le grand structuraliste américain L. Bloomfield (1926, p. 153): "The totality of utterances that can be made in a speech-community is the language of that speech-community." ${ }^{2}$ Cet ensemble est ouvert, non-fini, parce qu'il est toujours possible de construire une phrase plus longue à partir d'une phrase de longueur quelconque. , $^{3,4}$

4. La grammaire est alors ce qui, lexique à part ${ }^{5}$, permet de distinguer ce qui est dans $\mathrm{E}$ de ce qui n'y est pas : en d'autres mots, la langue en intension.

4'. La compétence d'un sujet parlant une langue est donc sa grammaire interne, ce qui lui permet de construire un nombre indéfini de phrases/d'énoncés distincts, et, quand il en entend de nouveaux, de les comprendre et de reconnaître lesquels sont bien formés, et lesquels ne le sont pas.

4". L'ensemble des phrases étant non-fini, la grammaire que construit le linguiste pour rendre compte de cette compétence est générative, au sens mathématique du terme: elle est censée énumérer potentiellement toutes, et rien que, les phrases de E. Les

1. Claude Hagège (1996) a inversé la valeur des termes 'énoncé' et 'phrase', mais la plupart des linguistes s'en tiennent à la description (bien sommaire !) proposée ici.

2. Voir plus tôt, en dehors des linguistes, Wittgenstein (1921) dans le Tractatus, §4.001 : "La totalité des propositions est le langage" ; on comprendrait mieux : 'une langue est la totalité de ses propositions' - où il faut cependant comprendre 'proposition' au sens logique (voir la note suivante).

3. Voir encore Quine 1953, p. 49, qui écrit explicitement: "Le grammairien [...] s'occupe de découvrir les limites de la classe $\mathrm{K}$ des suites signifiantes de la langue [...] Il n'y a apparemment pas de borne supérieure à la longueur des membres de K." (trad. fr.: 2003 p. 86).

4. Il est par ailleurs vrai que Chomsky a insisté sur le fait que des suites de mots peuvent être syntaxiquement bien formées tout en étant ininterprétables, cf. son exemple bien connu : Colorless green ideas sleep furiously - mais cela ne fait qu'ajouter un argument à cette idée que l'ensemble des phrases d'une langue (naturelle) est non-fini. Notons au passage que le linguiste Lucien Tesnière, mort en 1954, avait fait la même observation en écrivant : « $[u]$ ne phrase peut être sémantiquement absurde tout en étant structuralement parfaitement correcte. Soit en effet la phrase : Le signal vert indique la voie libre, qui a un sens raisonnable ; si j'en remplace tous les mots chargés de sens par les mots de même espèce qui les suivent immédiatement par ordre alphabétique dans le dictionnaire, j’aboutis à la phrase : Le silence vertébral indispose la voile licite, qui conserve le même schéma structural inattaquable, mais qui n’a plus aucun sens. » (éd. de 1969, pp. 41-42).

5. Du moins à partir des années 60, car dans le premier modèle de Chomsky, les items lexicaux étaient des symboles terminaux, et étaient donc introduits par des règles syntaxiques. 
différentes grammaires que proposent les linguistes sont donc des modèles, au sens où les physiciens (mais pas les mathématiciens!) emploient ce terme ${ }^{6}$.

5. Une théorie linguistique, dans cette perspective, consiste en un ensemble d'hypothèses sur ce que l'on peut s'attendre à trouver, et aussi à ne pas trouver, dans les grammaires des langues naturelles. Comme ces dernières varient, on aura des options ou « paramètres », si bien que, par idéalisation, en particulier en ce qui concerne le rythme d'acquisition, la « grammaire universelle » chomskyenne est l'état initial de l'enfant apprenant sa langue maternelle, l'acquisition consistant, outre l'apprentissage du lexique (ce qui est peut-être moins trivial qu'on ne pourrait le penser), à fixer la valeur ces paramètres pour associer correctement les mots ${ }^{7}$.

Rien de très concret n'a été dit jusqu'ici. Mais il faut signaler dès maintenant que même si l'on partage cette approche, bien des options restent possibles dans les domaines grammaticaux les plus divers. Je ne citerai ici que le cas du statut des morphèmes liés, c'est-à-dire des affixes ( préfixes, ou suffixes ) $)^{8}$ : en effet, pendant trente-cinq années environ, l'héritage structuraliste de Chomsky l'a poussé à considérer que les mots fléchis étaient construits en syntaxe, de même que les syntagmes ou groupes de mots le sont ( par définition ), puisque l'unité significative

6. Bien que l'on ait l'habitude d'associer les termes génératif et transformationnel, il sont conceptuellement disjoints : dans la perspective chomskyenne décrite ici, ce qui compte c'est que la grammaire soit, par définition, générative au sens mathématique du terme. Dès le milieu des années 1980, des modèles explicitement non-transformationnels ont été proposés comme alternative à l'approche chomskyenne stricto sensu, cf. G. Gazdar et al.., (1985), et bien d'autres travaux depuis. Inversement, de nombreuses grammaires font appel, d'un manière ou d'une autre, à la notion de transformation, et ce, depuis l'Antiquité (Baratin 1989) jusqu'aux travaux de Zellig Harris dans les années 1950, en passant par la Grammaire de Port-Royal, sans parler des grammaires pédagogiques. De plus, ces transformations se faisaient (ou se font à partir d'une structure de surface primitive en structure de surface dérivée, ce qui les distingue radicalement de la dérivation chomskyenne, qui construit des structures sous-jacentes d'où deux structures de surface en relation de paraphrase (par exemple quand il y a alternance entre voix active et voix passive sans quantification sur les arguments ou actants) sont précisément, l'une comme l'autre, dérivées tranformationnellement.

Enfin, il existe aussi des approches non pas dérivationnelles, mais «vérificationnelles», qui permettent de «calculer» si une phrase donnée est bien formée ou non, et appartient donc, ou non, à l'ensemble ouvert de phrases qui définit une langue; la première tentative dans cette direction est, à ma connaissance, celle de Y. Bar-Hillel (1953) - curieusement ignoré Dans Chomsky (1956). Un autre modèle, plus proche de l'approche chomskyenne, est fourni par les travaux issus de Sadock (1991). Enfin, parmi les vérificationnistes les plus proches de Chomsky, noter Brody (1995), et ses publications suivantes.

7. Ce point a souvent été mal compris : cette " grammaire universelle » ne contient pas uniquement des universaux ou invariants linguistiques; il a même pu être proposé, de manière sans doute un peu provocante, que les invariants proprement dits ne sont que des paramètres à une seule valeur, donc des paramètres particulièrement triviaux...

8. Il existe aussi des infixes, comme dans le cas de la marque de la pluralité de l'objet direct du verbe basque eduki 'tenir', dont les voyelles initiale (e-) et finale (-i) ne font pas partie du radical (de forme -uka- quand ce verbe est fléchi), cf. da-uka-t 'je le tiens', mais d-auzka-t 'je les tiens'. 
minimale est le morphème $e^{9}$, et n'est le mot que lorsqu'il n'est ni composé, ni dérivé, ni fléchi, et se réduit donc à un seul morphème ${ }^{10}$.

\section{Types de règles et types de déviance}

Il faut distinguer entre trois sens ou emplois du mot «règle». Il y a en effet des règles prescriptives, des règles descriptives, et des règles qui sont des instructions computationnelles.

Tous les linguistes sont d'accord pour dénoncer les règles prescriptives (celles qui sont enseignées à l'école, et/ou promues par diverses institutions comme les académies) comme ne respectant pas toujours la réalité (par exemple, quelle est la proportion de francophones natifs qui emploient spontanément le passé simple ou encore l'imparfait du subjonctif à toutes les personnes, et dans tous les contextes définis par l'Académie française ?), et les remplacent par les règles descriptives, c'est-à-dire des descriptions de régularités.

On peut illustrer la différence entre les deux types de règles comme suit.

(a) Considérons les trois verbes de sens très proche que sont : se souvenir, se remémorer, se rappeler ; le premier prend toujours un complément introduit par la préposition de, le second prend un objet direct, et, si la grammaire prescriptive exige un objet direct (l'absence de préposition) pour le complément du troisième, il existe de fait deux options dans la langue spontanée en ce qui le concerne ; la position du linguiste est alors simplement de signaler que la forme sans de est la seule qui soit officiellement admise par les puristes, sans plus...

(b) En basque, le verbe conjugué s'accorde automatiquement (en personne et nombre) avec son sujet, avec l'objet direct, s'il en a un, et, dans certains parlers (Outre-Bidassoa), automatiquement, et dans d'autres parlers (Iparraldean), optionnellement, avec l'objet indirect éventuel ; pour les locuteurs ordinaires de basque péninsulaire, les locuteurs des parlers continentaux font des «fautes » quand ils n'accordent pas le verbe avec son complément marqué au cas datif ; pour un linguiste, par contre, il s'agit simplement à nouveau de variation dialectale, que toute description objective se doit de mentionner, sans plus à nouveau.

(c) En basque encore, comme l'a bien montré Oyharçabal (1987), si les relatives ordinaires précèdent usuellement le nom qu'elles modifient, dans tous les dialectes et à toutes les époques, on trouve des textes dans lesquels elles le suivent (avec la marque de nombre et de cas à la fin de la relative : il ne s'agit donc pas d'appositives). La plupart

9. Dans la terminologie usuelle, le «morphème » est aussi bien lexical que grammatical (ou fonctionnel); dans la terminologie d'A. Martinet, ce mot est réservé au second type, celui de « lexème » correspondant au premier type.

10. Pour une défense de la position chomskyenne initiale, et donc structuraliste, dans le domaine de la conjugaison des verbes, et en rapport avec la notion d'universaux ou plutôt d'invariants linguistiques, du moins quand ce type de flexion est agglutinant, cf. par ex. Rebuschi (2010), avec, en particulier, des références au basque (et au swahili). 
des grammairiens ont ignoré ce phénomène, mais il est explicitement signalé par Gèze (1873, p. 74) pour le souletin (je modernise l'orthographe):

«On peut dire indifféremment : gizun ikhusi düdanak hori egin dü ou ikhusi düdan gizunak hori egin du. $»^{11}$

De même, Archu (1868, p. 151), donne les deux versions (également souletines) suivantes pour la phrase 'La maison que j'avais achetée était belle': Erosi nüen etxea eder zen, « ou » Etxe erosi nüena eder zen.

On peut encore ajouter à ces deux auteurs (cités par Oyharçabal) un texte plus récent, tiré de Gavel \& Lacombe (1937, p. 20, note 1):

« Dans l'exemple ci-dessus [Gure auzoaren1 [atzo2 hil3 den4] seme5az6 mintzo ginen7 'Nous parlions7 du6 fils5 de notre voisin2, qui est5 mort4 hier3 - p. 19] on pourrait ainsi rejeter avantageusement après le mot seme le membre de phrase atzo hil den [i.e. la proposition relative] et dire : gure auzoaren1 seme5 [atzo2 hil3 den4]az6 mintzo ginen7. Comme on le voit, la proposition relative serait ici reportée après le mot qui lui sert d'antécédent, et traité comme adjectif qualificatif, ne formant avec l'antécédent seme qu'une même agglutination, dont le dernier élément serait seul décliné. » [C'est moi, G.R. qui ai introduit les numéros en indice et les crochets.]

On le voit, les limites du grammatical et de l'agrammatical ne sont pas claires, car la plupart des locuteurs natifs interrogés par Oyharçabal rejetaient ce second type de relatives, alors qu'ils en produisaient eux-mêmes... (communication personnelle de B.O.)

Cela dit, dans une approche dérivationnelle ou computationnelle, comme celle de la GGT, les règles ont un tout autre statut, et ne sont pas directement « falsifiables » (au sens poppérien du terme) ou invalidables : c'est l'ensemble de ces règles ${ }^{12}$ qui, s'il est correctement construit bien sûr, permet, comme il a été dit ci-dessus, de dériver potentiellement toutes et rien que les phrases jugées comme bien construites par les sujets parlants.

11. Voici l'analyse et la traduction de la première phrase de Gèze (celle du type souvent receté aujourd'hui) et d'Archu (qui donne, comme la seconde de Gèze, l'ordre des mots standard) - je modernise l'orhtographe):

(a) Gizun ikhusi düd-an-a-k hori egin dü

homme vu je-l'ai-n-SG-ERG cela fait il-l'a

'Lhomme que j'ai vu a fait cela.'

(ERG: ergatif, cas du sujet ou agent des verbes transitifs à la voix active.)

(b) Erosi nüen etxea eder zen.

acheté je-l'avais maison-SG beau/belle était (trad. en fr. dans le texte cité)

(Voir la note 20 plus bas pour quelques indications sur la construction des propositions relatives en basque.)

12. C'est la thèse, bien connue en philosophie des sciences, dite de «Duhem-Quine» 
Noter cependant, d'une part, qu'il arrive souvent, trop souvent, que les locuteurs soient influencés par les règles prescriptives qui leur ont été enseignées, si bien qu'ils peuvent rejeter des formes et des constructions qu'ils emploient régulièrement (toute situation de test ou d'enquête linguistique met évidemment en jeu la performance, et celle-ci est influencée par des facteurs socio-culturels) — et c'est précisément ce qui est arrivé à B. Oyharçabal quand il a interrogé des bascophones sur la question de l'ordre relatif du nom modifié et de la proposition relative). D'autre part, et en partie en rapport avec ce qui précède, il n'est pas évident que l'ensemble E soit bien défini, plutôt que flou: il faudrait postuler, comme l'a fait Chomsky depuis les années 60, un locuteur idéal (bien spécial) qui vit dans une communauté (trop) homogène pour envisager que E soit défini.

J'ai dit : « bien spécial » et « trop homogène ». C'est parce qu'il y a toujours des variations sociales, géographiques, mais aussi stylistiques et de registre : on ne parle (normalement) pas à son chien comme à une vieille dame inconnue à qui on demande son chemin, ou à un gendarme qui vient de vous siffler pour excès de vitesse.

C'est donc une question importante qui se pose au linguiste que de savoir s'il doit « tout » prendre, ou sélectionner ce qui correspond à un registre particulier, et elle est sans solution autre que décisionnelle et arbitraire - ce qui a forcément pour effet de rendre toutes les grammaires formelles hypothétiques, mêmesi leur rôle heuristique n'est plus à démontrer...

\section{L'opposition compétence/performance}

La performance, au sens chomskyen, c'est donc la production et la compréhension d'énoncés au sens défini plus haut. Mais ce n'est pas l'absence de dimension pragmatique qui importe ici : c'est l'idée qu'au-delà des énoncés balbutiés, interrompus, des difficultés rendues possibles par des défaillances de mémoire à (très) court terme, etc., le fait même de parler implique(rait) que le locuteur ou l'interlocuteur utilise sa connaissance ainsi modélisée dans son activité langagière, c'est-à-dire son activité linguistique spontanée. (Noter de plus que le modèle de compétence dérivationnel chomskyen est officiellement neutre par rapport à l'activité cérébrale de production ou de compréhension : on va y revenir).

\subsection{L'exemple classique des relatives avec "center-embedding"}

Il y a des choses qui semblent très simples à produire, et qu'on ne peut pas itérer, alors même que la récursivité est centrale dans le langage humain — car l'ensemble E est, on l'a dit plus haut, non-fini, dans la mesure où l'on ne peut pas établir une liste close des phrases possibles dans une langue (vivante) quelconque.

L'exemple le plus souvent proposé dans les manuels est le suivant. Il est toujours possible de remplacer un nom propre, par un groupe de mots (qu'on appelle syntagme nominal) contenant un article, un nom commun et une relative qui permet d'identifier ladite personne. Par exemple, à partir de la phrase (1) ci-dessous, si (2) est vrai ou considéré comme tel, ou pourra paraphraser (1) par (3):

(1) $\quad$ Pierre $_{\mathrm{i}}$ pleure.

(2) $\quad$ Pierre $_{\mathrm{i}}=\left[\right.$ le garcon que Marie aime $_{\mathrm{i}}$

(3) [Le garcon que Marie aime $_{i}$ pleure.

Ajoutons maintenant (4):

(4) $\quad$ Marie $_{j}=$ [la fille que Jean ${ }_{k}$ déteste $]_{j}$ 
Va-t-on pouvoir remplacer Marie par cette dernière expression?

On peut le faire dans de nombreux contextes, mais ici, cela donne:

$$
\text { \#[Le garçon que [la fille que } \left.\text { Jean }_{\mathrm{k}} \text { déteste }\right]_{\mathrm{j}} \text { aime }_{\mathrm{i}} \text { pleure. }^{13}
$$

Peut-on considérer que cet énoncé ne peut pas être construit ? Cela paraît bien difficile, d'autant plus que si l'on inverse le sujet et le verbe dans la relative, comme avec la variante (6) de (2):

(6) $\quad$ Pierre $_{\mathrm{i}}=$ [le garcon qu'aime Marie $\left._{\mathrm{j}}\right]_{\mathrm{i}}$

on forme sans mal :

(7) [Le garcon qu'aime Marie $]_{i}$ pleure

et, à partir de là, avec (4) :

(8) [Le garcon qu'aime [la fille que Jean ${ }_{k}$ déteste $\left.]_{j}\right]_{i}$ pleure.

phrase tout à fait compréhensible.

Si on pratique l'inversion du sujet et du verbe dans le goupe nominal qui remplace Marie, soit maintenant (9) comme paraphrase de (4):

$$
\text { Marie }_{\mathrm{j}}=\left[\text { la fille que déteste } \mathrm{Jean}_{\mathrm{k}}\right]_{\mathrm{j}}
$$

On obtient alors :

(10) [Le garcon qu'aime [la fille que déteste $\left.\left.\operatorname{Jean}_{\mathrm{k}}\right]_{\mathrm{j}}\right]_{\mathrm{i}}$ pleure.

qui est peut-être un peu plus difficile à gérer, mais par contre la suivante, (11), me paraît tout aussi inaccessible que (5), alors même que je sais bien ce qu'elle pourrait signifier :

(11) \#[Le garcon que [la fille que déteste Jean $\left._{k}\right]_{j}$ aime $]_{i}$ pleure.

Ce dernier exemple est crucial, car il permet d'éliminer une hypothèse concernant l'inacceptabilité de (5), à savoir que si deux verbes conjugués se suivent, et que le dernier, comme dans tous ces exemples, est final, il doit être prononcé sur un ton descendant, l'autre devant par contre être prononcé sur un ton ascendant (haut continuatif pour les spécialistes).

13. Le dièse \# signale la déviance d'un énoncé pour lequel il n'y a, pour autant que l'on sache, pas d'explication strictement grammaticale. 
On pourrait ainsi penser qu'on « explique » (5), car le verbe du milieu, aime, est soumis à deux contraintes prosodiques contradictoires, celle de devoir être simultanément prononcé avec un ton descendant et un ton montant. La phrase ne serait pas prononçable, ce qui signifierait, moyennant certaines hypothèses supplémentaires, qu'elle n'est pas compréhensible non plus.

MAIS dans (11), seuls deux verbes se suivent en fin de phrase, tout comme dans (3) ! Et pourtant, elle est incompréhensible ${ }^{14}$.

Lidée est donc qu'en performance (au sens où l'on parle et/ou comprend effectivement), il y a des phrases que notre cerveau ne peut pas traiter, bien qu'elles soient parfaitement bien formées du point de vue de la compétence de locuteurs du français.

De telles considérations ont donc amené Chomsky à proposer qu'en production ou compréhension, à côté de la compétence grammaticale, nous disposons d'un analyseur syntaxique (parser en anglais), et que celui-ci ne peut pas traiter de telles phrases évidemment, aussi longtemps qu'on n'a pas proposé cet analyseur, on n'a rien dit, mais les travaux ont avancé dans cette direction, au moins depuis l'ensemble des textes regroupés dans Dowty et al. (éds.), 1985.

\subsection{Les inconstructibles}

On vient de voir que des opérations parfaitement naturelles (et respectant les contraintes liées à la structure de constituants) peuvent être itérées d'une certaine manière (en complexifiant éventuellement le résultat par inversion), et donner lieu à des énoncés parfaitement compréhensibles, mais ne le peuvent pas d'une autre manière, ce qui constitue un premier argument en faveur de la distinction entre compétence et performance. Mais il existe deux autres cas de figure pertinents, qui, à ma connaissance, ne sont guère avancés dans la littérature technique (voir cependant la note 17).

Voyons d'abord le premier cas (le second fera l'objet de la section 5.3), celui de phrases qu'on pourrait imaginer, suite à une généralisation naturelle, être constructibles, i.e. artificiellement produites, mais qui, au contraire de (5) et (14) ci-dessus, ne le sont pas du tout - alors qu'elles peuvent être comprises plus ou moins facilement. Considérons ainsi les exemples suivants :

$$
\text { Beaucoup de gens entrent là-dedans, et peu en sortent. }
$$

On peut bien sûr avoir une paraphrase dite impersonnelle de (12):

$$
\text { Il entre beaucoup de gens là-dedans, et il en sort peu. }
$$

14. Il en va de même avec la passivation, qui ne se contente pas de modifier l'ordre des mots, mais introduit des morphèmes supplémentaires, cf. Le garçon qui est aimé de la fille que Jean déteste pleure / Le garçon qui est aimé de la fille qui est détestée par Jean pleure, phrases qui, quoique lourdes, restent parfaitement compréhensibles. Noter à cet égard le passage suivant de Chomsky (1965, p. 198, note 8) : «Yngve fait la remarque importante que certaines transformations peuvent être utilisées pour alléger le coût perceptuel, ce qui suggère un argument intéressant concernant la raison pour laquelle les grammaires contiendraient des transformations. »Cela dit, curieusement, depuis une vingtaine d'années, Chomsky a introduit dans son « programme minimaliste » la complexité computationnelle comme facteur jouant de manière très importante dans la compétence - et pas seulement la performance. 
Maintenant, remplaçons la coordination par une construction comparative. La phrase (14), impersonnelle, permet de le faire :

(14) Il entre plus de gens là-dedans qu'il n'en sort.

On peut aussi avoir une paraphrase dite existentielle, qui commence par la séquence Il y a: Il y a plus de gens qui entrent là-dedans qu'il n'y en a qui en sortent.

Mais revenons à la tournure manifestée par (12). On peut effectivement commencer par:

(16) Plus de gens entrent là-dedans...

Mais comment continuer? Ni qui ni que ne sont possibles:

(17) Plus de gens entrent là-dedans *que/*qui n'en sortent.

On est obligé de recourir à la construction impersonnelle :

(18) Plus de gens entrent là-dedans qu'il n'en sort. ${ }^{15}$

Il s'agit bien en (17) d'une question d'agrammaticalité, liée au savoir implicite des locuteurs de français, qui leur interdit d'employer la conjonction que dans ce contexte.

La compétence est donc toujours la compétence dans une langue donnée, et, comme les grammaires varient, on peut s'attendre à ce qu'un équivalent de (17) soit possible dans une autre langue naturelle, et c'est bien ce que permet l'anglais, qui possède en effet une conjonction de subordination spécifiquement comparative et qui est compatible avec une construction partitive, than, ce qui n'est pas le cas du français, et la connaissance de cette conjonction fait partie de la compétence de tout locuteur anglophone:

15. Un autre argument, emprunté au même type d'exemple, milite à nouveau en faveur d'une compétence grammaticale distincte de la capacité de produire et comprendre en ligne / en temps réel. Il s'agit de la contrainte (quelqu'en soit le statut exact) qui interdit la présence côte à côte de deux en, l'un renvoyant à une expression quantifiée nominale (cf. Il en sort beaucoup des facultés, mais peu des grandes écoles, où en peut renvoyer à 'des étudiants'), et l'autre à la source du déplacement (cf. Il y entre plus de garçons qu'il n'en sort de filles, où y et en renvoient à un même lieu). A côté de (14), il est donc strictement agrammatical de construire : *Il entre plus de gens là-dedans qu'il n'en en sort (l'astérisque signale des formes agrammaticales, au contraire de \#), bien que si un étranger produisait cette phrase, tout francophone la comprendrait sans mal, au contraire de (5) ou (11) [Dernière minute : pour certains locuteurs, cette forme serait bien formée et/ou acceptable mais ce n'est absolument pas mon cas]. Mais si ces clitiques appartiennent à deux propositions, l'occurrence de deux en est parfaitement possible, comme dans (15) : ... qu'il n'y en a qui en sortent... 
Soulignons que, par contre, l'équivalent anglais de (5) est tout aussi incompréhensible à un anglophone que (5) ne l'est à un francophone : l'opposition entre le statut strictement agrammatical de (17), qui n'est pas contructible en français et le statut grammatical de (19), vs. le caractère radicalement incompréhensible de (5) et de son équivalent anglais - à quoi il faut ajouter la parfaite acceptabilité de (8) - permet d'étayer raisonnablement une forme de distinction entre compétence (connaissance grammaticale, normalement inconsciente) et performance, activité langagière « en ligne ».

\section{Deux modèles cognitifs}

Un des problèmes posés par la notion de compétence est sa neutralité (souvent soulignée par Chomsky) par rapport à la performance, i.e. à la production et la compréhension qui font appel à elle: si les mécanismes qui permettent de parler et de comprendre doivent en être distincts, il n'y a pas de raison que la production en soit plus proche parce que les modèles chomskyens successifs ont presque toujours été purement dérivationnels ${ }^{17}$. Mais qu'est-ce que cela signifie exactement quand on lit qu'il y a « une procédure générative qui fabrique des expressions linguistiques avec leurs propriétés pertinentes aux interfaces [conceptuointentionnelle d'une part, perceptuo-articulatoire de l'autre], et des systèmes de performance qui accèdent à ces instructions [=les propriétés d'interface] et sont employés pour interpréter et exprimer les pensées. » (Chomsky 2000, p. 29) ? La réponse était fournie deux pages plus haut : "Toute expression linguistique générée par le langage-I[nterne] inclut des instructions

16. L'anglais présente évidemment aussi des tours intellectuellement possibles, c'est-à-dire constructibles sur le papier, mais agrammaticaux. Un exemple récent a été proposé par J. Fodor dans Boeckx et al. (2011, p.202). Il s'agit de l'impossibilité réelle de pluraliser How tall a man is John?, 'Quelle est la taille de John ?', car *How tall men are John and Bill? est irrémédiablement mauvais. (Et pourtant, on peut ajouter qu'un autre élément qui peut précéder l'article indéfini singulier, such 'tel' est, par contre, compatible avec le pluriel, cf. Such a man is incredible / Such men are incredible 'un tel homme / de tels hommes est/sont incroyables(s)'.)

17. Quand la théorie chomskyenne des années 1960, et elle seule, proposait une "structure profonde » servant d'input à l'interprétation (sémantique) des phrases, les transformations ne menant qu'à la « structure de surface » qui servait elle-même d'input à leur « interprétation phonétique », on a pu, bien à tort, considérer qu'elle modélisait plus ou moins la production de formes linguistiques qui codaient un sens qui leur préexistait (l'étape ultime de cette démarche étant fournie par l'abolition de la distinction entre structure profonde et représentation sémantique, ce qui a constitué le programme de la " sémantique générative » des années 1970). Mais il faut rappeler que, dans les années 50, la dérivation construisait le sens (ainsi, l'introduction de la négation était une transformation optionnelle, mais ô combien cruciale sur le plan sémantique !), et que, suite à l'introduction des traces puis de la «Forme logique », dans les années 70 également, l'interprétation (sémantique) s'est retrouvée en fin de dérivation, et à quelques détails près (remplacement de l'hypothèse de la Forme logique par la théorie dite des phases dans le « programme minimaliste »), cela reste vrai aujourd'hui. 
pour les systèmes de performance dans lesquels le langage-I [=la compétence] est enchâssé. » (ibid., p. 27). En d'autres termes, il y a une forme de préexistence de droit et de fait de la compétence linguistique par rapport aux systèmes de performance. ${ }^{18}$

Mais il faut noter autre chose: après le premier cognitivisme, computationnel, donc formel et démonstratif, est apparu le (néo-)connexionnisme, qui repose sur beaucoup moins de formalisme, et qui est assez à la mode, pour des raisons évidentes, dans la plupart des travaux en intelligence artificielle. Il n'y a plus nécessairement de contradiction entre une approche computationnelle de la compétence, et une approche connexionniste de la production spontanée, nonréfléchie, donc de la performance, si du moins, au lieu d'« enchâsser » la compétence dans la performance (ou «l es systèmes de performance »), on les articule d'une manière souple. Citons à cet égard le travail de Clark (1990), qui définit d'abord le modèle cognitif classique de performance dans les termes suivants:

A cognitive model is classical if it has a processing-level description which bears a certain rather close relation to the structure of a standard competence theory. A standard competence theory posits a set of rules or principles of derivation defined for a class of structured, symbolic representations according to their form. The close Relationship involves (i) the explicit representation, in the processing-level description, of the structured representations over which the rules are defined, and it involves (ii) the explicit or tacit representation of those rules and principles themselves.

Lauteur introduit ensuite un second modèle de performance, le connexionnisme, et propose une véritable articulation entre la compétence (computationnelle) et la performance (connexionniste) [c'est moi, GR, qui souligne] :

[C]onsider our capacity to parse garden-path sentences like the horse raced past the barn fell. A rogue model of parsing might do something like this. We have on-line a quick and dirty connectionist network which can parse most of the sentences we encounter in daily speech. But it does not have the capacity (even in principle, subject to idealization) to parse a garden-path sentence. However, we also have (not on-line, but in the background) a classical symbolic parser [...] which can parse such cases. And when the quick and dirty network fails, this back-up comes on-line to save the day. This fits the phenomenology, in which the sentence at first looks like nonsense, then falls into place. In such a case the classical competence theory correctly describes the structure of the back-up system. But it does not describe the on-line system. If,

18. La pluralité des systèmes de performance est évoquée en détails dans Chomsky (1980, p.224), où sont opposées compétence et performance grammaticales (avec une subdivision entre comp. et perf. syntaxiques, et comp. et perf. sémantiques), et pragmatiques. De plus «[1]a compétence pragmatique inclut probablement ce que Paul Grice a appelé 'la logique de la conversation'. On pourrait dire que la compétence pragmatique place la langue dans le contexte institutionnel de son emploi, mettant en relation les intentions et buts [du locuteur] avec les moyens linguistiques à sa disposition. » (pp 224-225). 
in addition, we imagine that the classical back-up system was active in training up the network, the partial confluence of the two systems over a range of simple cases is rendered unsurprising.

Cette phrase, the horse raced past the barn fell, fameuse dans certains cercles, est due à Bever (1970). Le verbe raced peut s'y interpréter soit comme le prétérite de to race 'courir': 'courut' soit comme le participe passé à valeur passive de l'emploi transitif (sémantiquement factitif ou causatif) de ce verbe, à traduire maintenant par 'faire courir', d'où le p.passé 'couru'. Tout lecteur d'anglais confronté à cette phrase est surpris par le dernier mot, fell 'tomba', car la première interprétation est la suivante : 'Le cheval a dépassé la grange au gallop', si bien qu'il n'y a plus d'interprétation disponible pour fell, à moins de revenir en arrière et de réinterpréter [raced past the barn] comme une adjonction adjectivale ou participiale à horse (plutôt qu'un syntagme verbal comprenant un verbe intransitif et un adjoint cicconstanciel), d'où l'interprétation 'Le cheval [auquel on a fait dépasser la grange au gallop] est tombé'.

Cela dit, en fait, la valeur de tels exemples est douteuse, puisqu'elle relève d'une activité non purement linguistique, la lecture; en effet, à l'oral, si l'énoncé complet devait être prononcé, raced serait alors produit avec un ton haut continuatif, et non un ton bas terminal ; or l'on sait depuis au moins Aristote (Interpr., §1) que l'écrit n'est qu'une représentation (bien imparfaite!) de la langue véritable, qui est fondamentalement orale. Ces considérations n'affectent pas globalement la position de Clark, mais doivent rester à l'esprit non seulement quand on lit des travaux de psycholinguistique qui traitent spécifiquement de langue écrite (comme par ex. Ferreira et al. 2001), mais aussi et surtout quand on utilise ce genre de phrases écrites pour illustrer la distinction entre compétence et performance, ce que je me garde bien de faire ici et c'est la raison pour laquelle je n'ai pas utilisé ce genre d'exemple dans l'exposition orthodoxe de l'opposition entre compétence et performance supra.

Cela dit, que ce qui vaut pour la compréhension vaut également pour la production, ce que souligne le fait qu'il y a en quelque sorte deux manières de parler : la plus ordinaire est évidemment spontanée, et a recours au système connexionniste rustique, grossier (pour reprendre les mots de Clark), mais l'on a parfois besoin de rechercher non seulement ses mots, mais ses tours de phrases, auquel cas on ferait appel au système de compétence chomskyen (quelqu'en soit le modèle computationnel spécifique). La plupart des générativistes, aujourd'hui encore, et donc 25 ans après la publication, par exemple, de Dowty et al. (éds., 1995) ou de McClelland \& Rumelhart (éds., 1987), ignorent ce que Clark ne présente pas comme une alternative radicale, mais comme une seconde approche qui doit être associée au modèle de compétence computationnel originel.

\section{Deux compétences grammaticales distinctes?}

\subsection{Introduction}

Nous avons vu jusqu'ici, d'une part, l'exemple de structures a priori faciles à construire, mais qui ne sont pas produites par des locuteurs natifs, et qui sont de plus incompréhensibles, même avec du recul, comme l'ex.(5) supra: de telles constructions ne seraient pas accessibles à l'analyseur syntaxique (anglais : parser) mobilisé (en plus de la compétence dans la perspective chomskyenne) dans la compréhension « en ligne ». Nous avons également vu, d'autre part, des structures qui ne peuvent pas être construites, qui sont rejetées par les locuteurs du 
français auxquels on les présente, mais qui restent marginalement compréhensibles, cf. (17). Mais on peut encore imaginer un autre type de déviance : il y a des choses que la compétence grammaticale devrait bloquer, et que l'on peut cependant construire, sans qu'il s'agisse de balbutiement ou de tout autre travers de performance au sens ordinaire du terme.

Dans ce cas, on retrouve ce qui suit du dernier alinéa de la section précédente, à savoir, qu'il y a en quelque sorte deux compétences distinctes, celle employée dans le discours spontané (qui ne saurait évidemment être de la «performance pure », car cette expression n'a aucun sens), et celle employée dans le discours réfléchi, dans la compréhension qui requiert de la concentration - et que l'on mobilise aussi quand on est amené à se poser des questions de grammaticalité (par exemple en répondant à des questions posées au cours d'une enquête linguistique). Largument que je vais proposer ici (qui concentrera à nouveau les relatives en basque) repose sur l'existence manifeste d'une capacité à produire des formes morphologiques complexes, qui sont en rapport avec une structure syntaxique elle-même relativement complexe, et qui vont à l'encontre du sens de la grammaticalité qui constitue l'une des facettes de la compétence chomskyenne. Mais il faut d'abord donner quelques indications supplémentaires sur le fonctionnement du basque.

\subsection{Arrière-plan grammatical : l'accord relatif à distance}

En basque, quand l'élément modifié par une relative est éloigné de cette dernière parce qu'elle est enchâssée dans une proposition complétive intermédiaire, il est possible d'accorder l'auxiliaire fléchi de ladite complétive en nombre avec le nominal modifié par la relative ${ }^{19}$, antécédent qui n'est pourtant pas l'objet du verbe de cette complétive. C'est ce qu'illustre la paire (20-21), due à Oyharçabal (1987, p. 131), le premier linguiste à avoir décrit ce phénomène) :

zozoak dire-la erraiten du-zu-n gizon-ak ${ }^{20}$ stupides ils-sont-que disant vous-l('a)vez-que hommes-les 'les hommes que vous dites être stupides / au sujet desquels vous dites qu'ils sont stupides'

19. Il existe un autre type d'accord dit «à distance » en basque, qui se manifeste entre un verbe fléchi et le complément d'un verbe nominalisé, et donc non-fléchi : cf. Lafitte (1962 : p. 255, § 531), et Etxepare (2006). Comme il n'y a pas d'« opérateur relatif » dans ces structures-là, je ne me prononcerai par sur la possibilité éventuelle d'unifier le traitement de ce second type d'accord à distance avec celui décrit ici.

20. Pour les lecteurs qui ne connaissent pas le basque, précisons que le suffixe -n sur l'auxiliaire conjugué marque entre autres choses la subordination relative : c'est lui que l'on retrouve sur l'auxilaire qui accompagne le participe présent erraiten du verbe 'dire', car le verbe ainsi suffixé « doit » précéder le nom modifié — à la réserve près du phénomène décrit en section 2, (c). Un exemple plus simple serait le suivant, où il n'y a pas d'intervention du verbe 'dire' (qui requiert, par contre, le suffixe -la) :

(a) zozo-ak dire-n gizon-ak

stupide-PL sont-n homme-PL

'les hommes qui sont stupides'

Pour que ce qui suit soit mieux compris, ajoutons que l'ellipse du nom modifié (notée ici par un $\varnothing$ en gras) est parfaitement naturelle, cf. (b) :

(b) zozo-ak dire-n- $\emptyset$-ak

'ceux [lit. : les $\emptyset$ ] qui sont stupides' 
Lauteur insiste sur le fait que (21) est plus naturel que (20) pour de nombreux bascophones. En tout état de cause, on peut considérer que les deux phrases sont bien formées. Si certains locuteurs rejettent cependant comme agrammaticale une phrase comme (21), cela peut être soit parce qu'elle n'entre pas dans leur grammaire interne, soit qu'ils ont le sentiment, peutêtre induit par l'enseignement scolaire (?), que ce n'est pas «bien» de parler comme cela. ${ }^{22}$

Oyharçabal (2005) fournit un autre exemple, plus pertinent pour ce qui va suivre, car il implique aussi la relativisation du complément d'une postposition (position du complément indiquée ici par le tiret '-'), ainsi que l'absence d'une tête nominale explicite. Le voici :

(22) (Maitatu behar ditugu [...]) — kontra ditugula uste aimer nous devons contre que-nous-les-avons croyance d-it-u-gu-n-ak berak. (Jauretxe, 1840, p. 105) nous-les-avons-n-PL eux-mêmes

21. En français, on relativise plus facilement la position objet que la position de la relative insérée dans la complétive, cf. les divergences de jugement de nombreux locuteurs quant à (b), structure bien attestée dans la littérature classique):

(a) $\sqrt{ }$ Voici la fille [que je crois [que Pierre aime]]

(b) \% Voici la fille [que je crois [qui aime Pierre]]

Est-ce un problème de compétence, ou de performance?

22. Techniquement, depuis les années 1980, on postule l'existence d'un opérateur relatif abstrait (non-prononcé) Op qui monte dans le spécificateur de la projection CP (et poursuit naturellement son déplacement dans le cas de (20)-(21), déclenchant ainsi optionnellement l'accord pluriel dans (21). C'est d'ailleurs l'analyse proposée par Oyharçabal (2005). Cet opérateur porte, par hypothèse, outre le trait de nombre, le trait de personne de la position relativisée, puisque ce même type d'accord «à distance » se retrouve dans (b) ciaprès, où le verbe fléchi qui accompagne uste 'croyance' porte une marque d'objet direct de 2e personne (où le pluriel est de politesse), za-it- (vs. le d- par défaut de 3e p. sg. dans (a)) [je remercie Beñat Oyharçabal pour une discussion de ces exemples] :

(a) (Zuri,) [oso ona zirela uste d-u-d-an-a-ri], egia erranen dautzut.

à-vous très bon êtes-que croire je-l'ai-n-SG-à vérité-SG je-vous-la-dirai

'A vous qui êtes très bon, je dirai la vérité.'

(b) (Zuri,) oso ona zirela uste zait-u-d-an-a-ri, egia erranen dautzut.

id. 
'(Nous devons aimer) ceux-là mêmes que nous croyons être contre nous.' lit. '... ceux-là mêmes que nous les-croyons que nous les-avons contre.'23

\title{
5.3. Le «monstre » morpho-syntaxique de Koldo Mitxelena
}

Passons maintenant à un exemple plus directement pertinent pour la question de la nature de la compétence et de la performance. Un grand intellectuel basque, Koldo Mitxelena, qui a fait partie des promoteurs de l'euskara batua, et qui est souvent considéré comme un modèle pour la langue écrite en basque unifié ou standardisé, a produit une forme comme la suivante, le dernier mot de (23) - exemple cité déjà dans Oyharçabal (1987, p. 133) et Euskaltzaindia (1999, pp. 194-195): 24

\author{
Ez d-it-ut aurkitu bila nen-bil-tza-n-ak. \\ NÉG je-les-ai trouvé à-la-recherche je-!aller-les-passé+QUE ${ }^{25}$-PLUR \\ 'Je n'ai pas trouvé ceux que je cherchais.' \\ lit. '(Je ne les ai pas trouvé[s]) les-que-je-les-allais à-la-recherche.'
}

(23) est une expression elliptique (cf. la note 23, ex. (b)), car un nom fourni par le contexte, adibide, 'exemple' n'est pas répété; plus explicitement, on aurait, à partir de (24), la relative (25), qui répète l'accord relatif optionnel en nombre illustré par la paire (20)-(21), et où le tiret indique la position relativisée dans la subordonnée :

(24) [Adibide-en bila] nenbil-en. ${ }^{26}$ exemple-PL+GÉN à-la-recherche j'allais 'Je cherchais les exemples/J'étais à la recherche des exemples.'

23. Oyharçabal (2005) cite aussi un exemple réel, associé au verbe nahi izan 'vouloir' dans la proposition matrice, dont l'auxiliaire porte une marque d'objet direct pluriel, alors que l'élément relativisé n'est que l'objet du verbe egin 'faire' de la relative :

Gizonek zuei egin dietzazuen nahi dituzuen gauza guziak (egin ietzezue zuek-ere heihalaber)

(Leiçarrague, 1571 : Mat 7,12),

lit. 'Toutes les choses que vous-les-voulez que les hommes vous fassent, (faites-les-leur vous aussi à eux.)'

J'ai de mon côté trouvé trois autres traductions de Mat 7,12, du 19 siècle maintenant, qui pratiquent cet «accord relatif à distance »: (i) Salaberry [dit] d'Ibarrolle (1856) : Egizu beraz gizoner hek zuri egin dezazien nahi dituzun guziak) et (ii) Cazenave (ms. \pm 1855 ) : Izi beaz gizoner zihauk hetaik nahi tzien, pour le basque du Nord - on trouvera une confirmation de la pluralité de l'objet de tzien dans Yrizar 1999, tableau II-27-c: tzie 'vosotros los habeis'); (iii) enfin, il y a le ms. d’Udabe ( \pm 1855$)$ en guipuzcoan : gauza guztiak nai zenustekianak gizonak dizaten zuekin, ala zuek ere egin berakin (la version publiée à Londres par L.-L. Bonaparte a par contre été « corrigée » par ce dernier). Noter de plus que dans les deux versions continentales autres que celle de Leiçarrague, le verbe fléchi de la proposition radicale ne porte pas de marque de pluralité!

24. Loriginal est dans Mitxelena (1981, p. 70), et le commentaire ajouté plus tard par Mitxelena lui-même (et mentionné plus bas dans le texte) est dans la note 25 (p.80).

25. Les suffixes de passé (e/)-n et de subordination relative -(e)n se contractent en un seul.

26. Nenbilen est une forme du basque standardisé, fondée sur la morphologie guipuzcoane : en basque continental, on aurait nindabilan, ce qui ne change rien au phénomène qui nous intéresse. 
Enfin, avec le $\varnothing$ qui marque l'ellipse, voici l'analyse morpho-syntaxique de la partie cruciale de (23):

$$
\text { [- bila nenbilen/nenbil-(*)tza-n- } \varnothing \text {-ak }]
$$

Dans (23), (25) et (26) donc, le verbe intransitif ibili ${ }^{27}$ contient une marque de pluriel, -tza, en plus de son accord normal avec le sujet de lère personne du singulier, nen-. Dans une note de bas de page, rédigée après la première rédaction de l'article dans lequel cette forme apparaît, Mitxelena parle d'un «monstre épouvantable » (ikaragarrizko mustroa) qui lui est «venu spontanément » (ustekabean itzuri zitzaidan), ce qui signifie que sa compétence (au sens chomskyen) ne lui permettait pas de produire un tel mot.

Si donc il a pu créer spontanément bila nenbiltzanak, et puis ensuite trouver que c'était un monstre abominable, c'est parce que, dans un premier temps, les mécanismes « rustiques » qui correspondent par définition au modèle connexionniste lui ont permis de le faire, puis, dans un second temps, parce que le recul que lui a donné la lecture des épreuves, recul qui accède à la compétence computationnelle chomskyenne (qui est en arrière plan selon Clark), a été activé.

Ajoutons quelques mots sur la morphologie verbale du basque pour comprendre comment nenbiltzan a pu être construit. Il faut d'abord noter que l'accord des verbes avec plusieurs arguments n'est pas une rareté typologique: de l'eskimo aux langues australiennes en passant par les langues uto-aztèques et maya d'Amérique centrale, la plupart des langues du Caucase et les langues bantoues d'Afrique, il en existe partout à la surface du globe. Plus rare sans doute est le fait qu'une marque de pluriel soit systématiquement distincte de la marque de personne proprement dite. Ainsi, autour du radical $-u$ - du verbe 'avoir', on opposera non seulement $d_{i}-u-t_{j}$ 'jej li'ai' à $d_{i}-i t_{k}-u-t_{j}$, où -it- marque le pluriel du complément : 'je $e_{j} l e_{i} s_{k} a i$ ', ou les deux formes, $d-u-z u$ et $d$-it-u-zu de (21) et (23) respectivement, mais aussi, par exemple, $n a_{i}-u-z u_{j}$ 'vous $\mathrm{j}_{\mathrm{i}}$ 'avez' à $g a_{i}-i t_{k}-u-z u_{j}$ 'vous ${ }_{j}$ nous avez' où le préfixe gai- identifie pourtant de manière non-ambiguë la première personne du pluriel, et où le second préfixe, -it-k, peut donc être considéré comme redondant ; voir encore, entre autres, n-oa 'je vais', et g-oa-z / g-oa-tzi 'nous allons', ou, avec ibili 'aller, marcher, s'occuper' qui apparaît dans (23)-(26): na-bil (a) 'jei

27. Il existe un emploi relativement rare de ibili transitif (comparer e-ra-bili 'utiliser', lit. 'faire fonctionner'). En voici un exemple- (c'est un vers du poète bas-navarrais Xalbador (1920- 1976), cité dans Aranalde (2001, p. 100):

Gau eta egun hitz hok gogoan da-bil-tza-t

nuit et jour mot ces dans-l'esprit je-les-'marche'

'Jour et nuit je retourne ces mots dans ma tête.'

Dans cet exemple, la conjugaison d'ibili est transitive : le préfixe d(a)-indique un absolutif (l'objet direct) de $3^{\text {e }}$ personne, le premier suffixe, -tza-, la pluralité de cet objet, et le second, - $t$, un sujet/agent de $1^{\text {ère }} \mathrm{p}$. du singulier. C'est donc quelque chose de parfaitement régulier. 
marche' mais $g a_{i}$-bil-tz(a) 'nousi marchons'. Le suffixe pluralisateur - $t z(a)$ est donc disponible avec ibili (de même que -it- l'est avec ukan/izan 'avoir', et -z/-tzi avec joan 'aller'), qu'il s'agisse de marquer la pluralité du sujet intransitif dans galbiltza, ou celle de l'objet direct dans la forme dabiltzat de la note $26^{28}$.

Si l'on ajoute à cette disponibilité de marque de pluriel absolutif la possibilité depratiquer l'accord relatif exhibé par l'auxiliaire à marque de pluriel $d$-it-uzu-n de (21), on voit que la « monstruosité » du nenbiltzan de (23) n'est que le fruit de l'application de ce qui conduit de la forme conjuguée de (20), d-u-zu(-n) à la forme de (21), d-it-u-zu(-n) avec, bien sûr le malaise intellectuel provoqué par l'inévitable réanalyse ou prise de conscience, due au recul de la relecture, de la forme nen-bil-tza-n comme comportant simultanément un préfixe d'accord absolutif singulier (avec le sujet intransitif) et un suffixe censé marquer de manière redondante (par ex. dans le cas de ga-bil-tz(a) cité à l'instant) le pluriel de l'argument déjà dénoté par le préfixe de la forme verbale conjuguée, et qui ne peut ici correspondre à aucun autre argument du verbe. Enfin, ce morphème -tz(a) permet de récupérer l'information que l'élément phoniquement vide à gauche de bila est au pluriel, de même que -gu- le fait régulièrement dans la première occurrence de ditugunak dans l'ex. (22).

En résumé, si accorder un verbe intransitif avec deux arguments absolutifs distincts est pour le moins une étrangeté, voire une "monstruosité », elle n'est pas apparue n'importe comment. Cela dit, le scénario réel de la production spontanée de cette forme, suivie plus tard du jugement de profonde anormalité, illustre parfaitement, à mon avis, la position de Clark (1990) : Mitxelena a construit la forme nenbiltzan spontanément, « en ligne », sans laisser le temps à sa compétence d'arrière-plan de bloquer cette forme.

Qui plus est, la construction même de nenbiltzan constitue un argument de choix pour défendre l'idée que, au moins quand la morphologie flexionnelle est agglutinante, les mots fléchis sont construits « en ligne », plutôt que tirés d'un lexique qui les contiendrait tous ${ }^{29}$ :

28. Rappelons que la morphologie active ou ergative du basque revient essentiellement à traiter de la même manière le sujet des verbes intransitifs (ou du moins de leur sous-ensemble dit « inaccusatif ») et l'objet direct des verbes transitifs, l'agent ou sujet des verbes transitifs étant traité à part (cas et accord dits ergatifs).

29. Dans les modèles de compétence chomskyens proposés des années 1950 à la fin des années 1980, la morphologie flexionnelle était construite syntaxiquement, Chomsky ayant en effet suivi durant cette longue période la tradition structuraliste en prenant le morphème (plutôt que le mot) comme unité syntaxique (cf. la fin de la section 1 supra) : ce n'est que dans son programme minimaliste qu'il a changé de position (sans guère de discussion d'ailleurs), en proposant que, dans la dérivation computationnelle, les mots soient tirés entièrement fléchis du lexique.

Ajoutons que la pléthore de variantes des formes verbales fléchies du basque constitue un argument (intuitif il est vrai) en faveur d'une création spontanée de formes. Outre les travaux d'Yrizar (dont un seul livre est cité ici, mais qui a passé en revue tous les dialectes), on peut citer encore Aurrekoetxea et al. (2005), qui utilisent un corpus qu'Yrizar n'a pas exploité ; pour ne prendre qu'un exemple emprunté à ce travail, sur cinq communes adjacentes où se parle le «même » (sous-) dialecte, le bas-navarrais occidental, et qui se suivent du nord au sud le long de la frontière avec l'Espagne, à savoir : Bidarray, Saint-Etienne de Baïgorry, Banca, Les Aldudes et Urepel, on peut constater qu'on n'en trouve pas deux qui partagent les mêmes formes conjuguées pour l'auxiliaire standardisé -iezaiozue (impératif à agent de $2^{\mathrm{e}} \mathrm{p}$. pl., objet direct et objet indirect

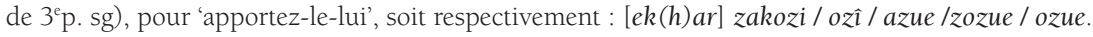


personne en effet ne peut imaginer que cette forme ait été ou soit stockée quelque part dans le cerveau/esprit de quelque bascophone que ce soit.

\section{En guise de conclusion}

Nous avons examiné différents types de déviances en français et en basque par rapport à une norme - dont le statut incertain et souvent fluctuant a bien dû être reconnu. Il y a eu d'abord l'argument standard - et inattaquable - avancé en faveur de la possibilité théorique et pratique (sur le papier) de construire certaines expressions et phrases qui s'avèrent incompréhensibles pour des raisons qui semblent effectivement n'avoir rien à voir avec les mécanismes normaux de complexification des expressions linguistiques par itération, ce qui constitue une justification indéniable pour l'établissement d'une distinction entre la (ou: une certaine forme de) compétence et la performance.

On y a ajouté un cas d'inconstructibilité syntaxique en français, qui s'oppose directement à l'anglais, ce qui montre que toute compétence linguistique est une compétence dans, ou pour, une langue donnée.

Enfin, on a abordé les questions soulevées par la construction, par un linguiste basque fort avisé, d'une forme «monstrueuse », qu'il a ultérieurement reconnue comme telle et qu'il a lui même caractérisée de cette manière-là : on a montré qu'elle correspondait dans tous ses détails à l'utilisation justifiée indépendamment d'un morphème de pluralité individuellement attesté, employé de manière normale dans des contextes syntaxiques très proches (l'accord relatif à distance), et qui, au bout du compte, permet d'identifier le nombre d'un pivot de relativisation, le seul défaut de cette forme étant que ce suffixe de pluriel apparaît sur un verbe intransitif qui est marqué pour un sujet au singulier ${ }^{30}$.

30. Comme il a été dit dans la note 22, on postulerait l'existence d'un opérateur relatif abstrait Op qui monterait dans le spécificateur de la première projection CP, puis poursuivrait son déplacement dans le cas de (20)(21), déclenchant ainsi optionnellement l'accord pluriel de (21). Ce qui rend (21) grammatical - par rapport à (23) - , c'est que le verbe lexical qui accompagne l'auxilaire qui porte la marque d'accord relatif à distance est transitif, et que son complément, plutôt que d'être une expression nominale, est une proposition complétive, qui n'a pas de marque casuelle : dans l'auxiliaire donc, la case, ou position, ou slot d'absolutif reste donc disponible, et peut être mise en relation avec une marque de nombre pour un autre élément. Dans le cas de (22), il n'est pas difficile de poser que la suite uste izan/ukan lit. 'croyance avoir' est réanalysée comme un verbe complexe, sans objet direct nominal non plus donc, si bien que l'on retrouve le cas de figure de (21). Ces hypothèses ne peuvent évidemment s'étendre à l'auxiliaire intransitif nen-bil-en/*tz-an, qui se distingue minimalement de la forme transitive avec sujet-agent de $1^{\mathrm{e}} \mathrm{p}$. du singulier et objet de $3^{\mathrm{e}} \mathrm{p}$. du pluriel au passé : ne-bil-tz-an).

Noter encore qu'outre le trait de nombre, l'opérateur relatif porte aussi le trait de personne de la position relativisée, puisque ce même type d'accord « à distance » se retrouve dans (b) ci-après, où le verbe fléchi qui accompagne uste 'croyance' porte une marque d'objet direct de $2^{\mathrm{e}}$ personne (où le pluriel est de politesse), za-it- (vs. le d- par défaut de $3^{\mathrm{e}} \mathrm{p} . \mathrm{sg}$.dans (a)) [je remercie Beñat Oyharçabal pour une disussion de ces exemples]:
(a) (Zuri,) [oso ona zirela uste d-u-d-an-a-ri], egia erranen dautzut.
à-vous très bon êtes-que croire je-l'ai-n-SG-à vérité-sg je-vous-la-dirai
'A vous qui êtes très bon, je dirai la vérité.'
(b) (Zuri,) oso ona zirela uste zait-u-d-an-a-ri, egia erranen dautzut.
id. 
Si toute production d'énoncés doit accéder à la compétence au sens chomskyen du terme, on ne voit pas comment Koldo Mitxelena aurait pu produire cette forme étrange. Il faut donc postuler l'existence d'une capacité proprement linguistique (car les objets en jeu, des marques d'accord, sont purement grammaticaux) à construire spontanément, « en ligne », pour reprendre les mots d'Andy Clark, des mots fléchis en rapport avec le contexte syntaxique large, capacité qu'il faut donc bien distinguer de la compétence chomskyenne, laquelle existe aussi bien entendu, puisque K.M. a étiqueté ultérieurement la forme $\left(^{*}\right)$ nenbitzan comme monstrueuse.

\section{Bibliographie}

\section{Sources des exemples basques cités}

ARANALDE, J.M., 2001. Xalbadorren barrua. Oyarzun, Sendoa (Auspoa liburutegia).

CAZENAVE (Abbé -) [ \pm 1860$]$, Jesu Khristoin Ebanjelio Seindia San Mathiuin aäbera. Ms., publié in R. M. Pagola et al. (éds.), Bonaparte Ondareko Eskuizkribuak: Ekialdeko Behenafarrera, I (Bilbao, Deustuko Unibertsitatea, 1999), 17-76.

JAURETCHE (Abbé -). 1840. Meditacioneac urtheco Ebanjelioen gainean [...]. Bayonne, Cluzeau.

LEIZARRAGA [L(E)IÇARRAGUE], J., 1571. Iesus Christ Gure Iaunaren Testamentu Berria

- Kalendera - ABC edo Christinoen Instructionea. La Rochelle. Facsim. sous le titre

I. Leiçarragas Baskische Bücher von 1571,éd. par Th. Linschmann \& H. Schuchardt, Strasbourg, 1900. Facsim. de ce dernier, Bilbao, Euskaltzaindia, 1990.

MITXELENA, K. [MICHELENA, L.], 1981.'Galdegaia eta mintzagaia euskaraz'. In : Euskal linguistika eta literatura: Bide berriak (Bilbao, Deustuko Unibertsitateko Argitarazioak), 57 81 .

SALABERRY, M., dit d'Tbarrole, 1856. L'Evangile selon Saint Mathieu, sur la version de M. le Maistre de Sacy [...]. Bayonne, Veuve Lamaignère. UDABE [Aita/Padre —] [ms.: 1856] 1993. Evangelio Santu gure Jesu Cristo Jaunarena, Done Matheoren arauran [...]. In : M.A. Pagola et al. (eds.), Bonaparte Ondareko Eskuizkribuak, Gipuzkera, IV (Univ. of Deusto, Deiker), 1969-2024. [Version corrigée par L.-L. Bonaparte des 7 premiers chapitres et du début du 8e : publiée à Londres en 1857, et reproduction facsim. in: L.L.Bonaparte, Opera Omnia Vasconice IV, Bilbao, Euskaltzaindia, 11-29.]

\section{Autres références}

ARCHU, J.-B., 1868, Grammaire bilingue, fançaise et basque - Bi mihiren gramatika, uskara eta franzesa, Bayonne, Lasserre. Rééd. : Hordago-Lur (Euskal Klasikoak 19), 1979.

AURREKOETXEA, Gotzon, VIDEGAIN, Xarles, \& IGLESIAS, Aitor. 2005. 'Bourciez' bildumako euskal atlasas (BBEA), 2 : Gramatika. ASJU 39.1, 1-277 (volume complet).

BARATIN, M. 1989. Naissance de la syntaxe à Rome. Paris, Minuit, 1989.

BAR-HILLEL, Y. 1953. 'A Quasi-Arithmetical Notation for Syntactic Description'. Language 29.1, 47-58 [Trad. fr. : 'Une notation quasi-arithmétique destinée aux descriptions syntaxiques', Langages 9, 1968, 9-22].

BEVER, T.G., 1970. 'The Cognitive Basis for Linguistic Structures'. In : J. R. Hayes, (éd.), 
Cognition and the Development of Language (New York, Wiley), 279-362.

BLOOMFIELD, L., 1926. 'A Set of Postulates for the Science of Language', Language 2.3, 153-164 [réédité in : M. Joos, (éd.), Readings in Linguistics, Washington, American Council of Learned Sciences, 1957].

BOECKX, C., FODOR, J.D., GLEITMAN, L. \&, RIZZI, L., 2009. 'Round Table: Language Universals: Yesterday, Today, and Tomorrow'. In : Piattelli-Palmarini M., Uriagereka J., \& Salaburu, P. (eds.), Of Minds and Language: A dialogue with Noam Chomsky in the Basque Country (Oxford, OUP), ch. 14, 195-220.

BRODY, M., 1995. Lexico-Logical Form. A radically minimalist theory. Cambridge (Mass.), MIT Press.

CHOMSKY, N., 1956. 'Three models for the description of language'. IRE Transactions on Information Theory 2.1, 113-124. [Trad. fr. dans Langages 9, 1968, 51-76.]

— 1965. Aspects of the Theory of Syntax. Cambridge (Mass.), MIT Press. [Trad. fr.: Paris, Seuil, 1971.]

_ 1980. 'Language and Unconscious Knowledge'. In : N. Chomsky, Rules and Representations (Oxford, Blackwell), 216-254. [Trad. fr. dans Règles et représentations, Paris, Payot, 1985]. Original du chapitre in : J. H. Smith (ed.), Psychoanalysis and Language, Psychiatry and the Humanities, 3, Yale University Press.

- 2000. New Horizons in the Study of Language and Mind. Cambridge, CUP.

CLARK, A., 1990. 'Connectionism, Competence, and Explanation'. The British Journal for the Philosophy of Science. Repris dans M. A. Boden (éd.), 1990, The Philosophy of Artificial Intelligence (Oxford, OUP, 1990), 280-308.

DOWTY, D.R., KARTTUNEN, L. \& ZWICKY, A.M., (éds.), 1985. Natural Language Parsing: Psychological, computational, and theoretical perspectives. Cambridge, CUP.

ETXEPARE, R., 2006. 'Number Long Distance Agreement in Substandard Basque'. In : J.A. Lakarra \& J.I. Hualde (éds.), Studies in Basque and Historical Linguistics in Memory of R.L. Trask - R.L. Trasken oroitzapenetan ikerketak euskalaritzaz eta hizkuntzalaritza historikoak, ASJU 40, 1-2, 303-350.

EUSKALTZAINDIA [Académie de la langue basque], 1999. Euskal Gramatika, Lehen Urratsa V: Mendeko perpausak (I). Bilbao, Euskaltzaindia.

FERREIRA, F., CHRISTIANSON, K. \& HOLLINGWORTH A., 2001. 'Misinterpretations of Garden-Path Sentences: Implications for models of sentence processing and reanalysis'. Journal of Psycholinguistic Research, 30.1, 3-20.

GAVEL, H. \& LACOMBE, G., 1937. Grammaire basque, tome II : Le verbe. Bayonne, Imprimerie du Courrier.

GAZDAR, G. et al., 1985. Generalized Phrase Structure Grammar. Oxford, Blackwell.

GĖZE, L., 1873. Éléments de grammaire basque (dialecte souletin), Bayonne, Veuve Lamaignière.

HAGÈGE, C. 1996. Lhomme de parole. Paris, Fayard.

LAFITTE, Pierre. 1962. Grammaire basque (navarro-labourdin littéraire). Bayonne, Editions des Amis du Musée basque \& Ikas.

McClelland, J. L. \& D. E. RUMELHART (eds.), 1987. Parallel Distributed Processing, Exploration in the Microstructure of Cognition, Vol. 2 : Psychological and Biological Models. Cambridge (Mass.), MIT Press.

MITXELENA, K., [Michelena, Luis] 1981. 'Galdegaia eta mintzagaia euskaraz'. In : Euskal 
linguistika eta literatura: Bide berriak (Bilbao, Deustuko Unibertsitatea), 57-81.

OYHARÇABAL, B., 1987. Etude descriptive de constructions complexes en basque [...]. Thèse d'Etat, Université Paris 7.

2005. 'Tokiz kanpoko numero komunztadura'. In : H. Knörr (éd.), Nerekin yaio nun. Txillardegiri omenaldia (Bilbao, Euskaltzaindia, Iker-17), 395-408.

QUINE, W.V.O. 1953. 'The Problem of Meaning in Linguistics'. In : From a Logical Point of View, Harvard Univ. Press, 47-54. [Trad. fr. : 'Le problème de la signification en linguistique', dans Du point de vue logique, Paris, Vrin, 2003.]

REBUSCHI, G., 2010. 'Sur la typologie des conjugaisons simples et composées'. In : I. ChoiJonin, et al. (éds.), Typologie et comparatisme. Hommages offerts à Alain Lemaréchal (Louvain, Peeters), 375-395.

SADOCK, J. 1991. Auto-lexical syntax: A theory of parallel grammatical representations. Chicago, University of Chicago Press.

TESNIÈRE, L., [†1954], 1969. Eléments de syntaxe structurale. Paris, Klincksieck (2 ${ }^{e}$ édition).

WITTGENSTEIN, L., 1921. Tractatus logico-philosophicus. Trad. fr. : Paris, Gallimard 1961.

YRIZAR Pedro de, 1999. Morfología del verbo auxiliar bajo-navarro oriental (Estudio dialectológico). Tomo I : Subdialecto Cizo-mixano. Bilbao, Euskatzaindia \& Universidad del País Vasco. 